\title{
The Influence of Inpatient Physician Continuity on Hospital Discharge
}

\author{
Carl van Walraven, MD MSc FRCPC
}

Medicine and Epidemiology \& Community Medicine, University of Ottawa, ICES uOttawa, Ottawa Hospital Research Institute, Ottawa, ON, Canada.

BACKGROUND: Inpatient attending physicians may change during a patient's hospital stay. This study measured the association of attending physician continuity and discharge probability.

METHODS: All patients admitted to general medicine service at a tertiary care teaching hospital in 2015 were included. Attending inpatient physician continuity was measured as the consecutive number of days each patient was treated by the same staff-person. Generalized estimating equation methods were used to model the adjusted association of attending inpatient physician continuity with daily discharge probability.

RESULTS: 6301 admissions involving 41 internists, 5134 patients, and 38,242 patient-days were studied. The final model had moderate discrimination (c-statistic $=0.70$ ) but excellent calibration (HosmerLemeshow statistic 11.5, $18 \mathrm{df}, p$ value 0.89). Daily discharge probability decreased significantly with greater severity of illness, higher patient death risk, and longer length of stay, on admission day, for elective admissions, and on the weekend. Discharge likelihood increased significantly with attending inpatient physician continuity; daily discharge probability increased for the average patient from 15.3 to $20.9 \%$ when the consecutive number of days the patient was treated by the same attending inpatient physician increased from 1 to 7 days.

CONCLUSIONS: Inpatient attending physician continuity is significantly associated with the likelihood of patient discharge. This finding could be considered if resource utilization is a factor when scheduling attending inpatient physician coverage.

KEY WORDS: hospital discharge; general internal medicine; continuity of care; generalized estimating equations.

J Gen Intern Med 34(9):1709-14

DOI: $10.1007 / \mathrm{s} 11606-019-05031-5$

(c) Society of General Internal Medicine 2019

\section{INTRODUCTION}

Physicians usually have time commitments other than patient care, including administrative, teaching, research,

Received February 6, 2018

Revised May 30, 2018

Accepted April 2, 2019

Published online June 13, 2019 and familial duties. Due to the nature of inpatient work, physicians often must change during a patient's hospitalization to allow for physician recovery time, to conduct other work (such as administrative, teaching, and research duties), and for personal obligations. In addition, inpatient medicine can be both physically, intellectually, and emotionally taxing necessitating time away from the ward and switching of inpatients between physicians. This switching results in fragmented physician assignment and decreased inpatient continuity of care.

The influence of attending inpatient physician continuity of care on inpatient outcomes has been evaluated in only a handful of studies. Most of these have focused on the influence of weekend cross-coverage on discharge probability. The randomized trial by Ali et al. found no differences in intensive care length of stay with weekend cross-coverage but differences in continuity between the "high-continuity" and "regular care" patients was very small. ${ }^{1}$ On the general medical ward, Epstein et al. studied patients with pneumonia and congestive heart failure and found that patients with greater cross-coverage (defined as the proportion of hospital days in which the patient was treated by a physician other than the primary) had significantly longer lengths of stays. ${ }^{2}$ The authors noted, however, that they were unable to determine whether increased LOS actually caused the increased crosscoverage rather than being a function of it. Blecker et al. ${ }^{3}$ addressed this issue of potential reverse causality by starting patient observation on the patient's first weekend in hospital. They found that the adjusted number of days in hospital (following the first weekend) was increased by more than $50 \%$ when patients had weekend cross-coverage. This study excluded observation time prior to weekends. A study from our center which included all patient time in hospital found that patients were more likely to be discharged from hospital as the consecutive number of days that the attending physician had been on service. ${ }^{4}$ However, it was unclear from this analysis whether the increased discharge probability was due to increased continuity of care or increased physician proficiency over time.

To determine whether attending inpatient physician continuity of care on general medical services influenced hospital length of stay, this current study measured the independent influence of physician continuity of care on the adjusted daily discharge probability. 


\section{METHODS}

\section{Study Setting and Databases Used for Analysis}

The study took place at The Ottawa Hospital, a 1000-bed teaching hospital with two campuses that is the tertiary referral center in our region. The division of general internal medicine has six patient services (or "teams") at two campuses led by an attending general internist, a senior medical resident (2nd year of training), and various numbers of interns and medical students. Attending inpatient physicians take care of only one patient service at a time (even on the weekends). With a few exceptions, all attendings also had varying degrees of ambulatory care duties.

Patients are admitted to each general internal medicine service (and each attending inpatient physician) on a daily basis almost exclusively from the emergency room. Patient assignment is essentially random since all services have the same clinical expertise (i.e., no services in the general medicine division have distinct clinical expertise influencing the service to which a particular patient might be preferentially admitted). The number of patients assigned daily to each service is usually equivalent between teams at a particular campus, although this can vary slightly (for example, a service might be skipped patient assignment if their total numbers are high or if they have challenges with house-staff coverage). Patients essentially never switch between teams but may be transferred to another clinical service. The study was approved by our local research ethics board.

All datasets used for the study reside within the Ottawa Hospital Data Warehouse. The Patient Registry Database records for each patient the date and time of admission (defined as the moment that a patient's admission request is entered into the database). Disposition is recorded, including death or discharge from hospital (defined as the time when the patient's death or discharge from hospital was entered into the database) as are transfers to other specialties. Emergency room encounters were also identified in the Patient Registry Database along with patient age and sex, and clinical service and location throughout the hospitalization. The Laboratory Database records all laboratory tests and their results.

\section{Study Cohort}

The Patient Registry Database was used to identify all patients admitted to the general medicine service between 1 January and 31 December 2015. This time frame was selected to ensure that data were complete. The general medicine service was studied because it is the largest inpatient specialty in the hospital.

\section{Study Outcome}

The primary study outcome was discharge from hospital as determined from the Patient Registry Database. Competing events included death in hospital and transfers to other specialties.

\section{Study Covariables}

The primary exposure variable was the consecutive number of days that a patient had been treated by the same attending inpatient physician. This exposure variable was timedependent (i.e., its value changed each day the patient was in hospital) and was measured at the patient-level (i.e., its value was unique to each patient on each hospital day). It is also worth noting that these values would vary between patients on a particular day for a particular inpatient physician based on how long they had been in hospital and whether they had been with that inpatient physician throughout their admission. When the inpatient physician changed for a particular patient, this number was reset to one.

Attending inpatient physician assignment to each general medicine service was determined from the call schedules for 2015. These call schedules are accurate because they are used by hospital locating to page physicians and also contribute to physician remuneration. Changes in inpatient physician and house-staff coverage are not concurrent. House-staff continuity was not measured.

Several other covariables were offered to the model. These included the expected daily probability of discharge measured with the TEND model. ${ }^{5}$ This used survival-tree methods to cluster together patient-days having similar daily discharge probabilities through patient factors (age, Laboratory Abnormality Physiological Score [LAPS] $]^{6}$ calculated at admission) and hospitalization factors (hospital campus and service, admission urgency, day of the week, ICU status). Within each cluster, the discharge probability on each hospitalization day was measured using data from people within that cluster over the previous 6 months. In a validation population, the expected number of daily discharges strongly predicted the observed number of discharges with an adjusted $R^{2}$ of $89.2 \%(p<0.0001)$ and a median relative difference between observed and expected number of discharges of only $1.4 \%$ (IQR -5.5 to $7.1 \%$ ). For the current study, expected discharge probabilities were calculated using a 6-month "look-back" of general medicine patients alone (rather than patients from all hospital services).

Other covariates offered to the model included the expected probability of death within a year of admission to hospital from the HOMR-now! model. ${ }^{7}$ This model used routinely collected data available at patient admission regarding the patient (sex, lifetable estimated 1-year death risk, Charlson score, current living location, previous cancer clinic status, and number of emergency department visits in the previous year) and the hospitalization (urgency, service, and LAPS) to predict the expected risk of death within 1 year. The model explained more than half of the total variability in death likelihood (Nagelkerke's $R^{2}$ value of 0.53 ), was very discriminative (c-statistic 0.92 ), and accurately predicted death risk (calibration slope 0.98 ). The daily LAPS ${ }^{6}$ was used as a measure of acute illness throughout the hospitalization. The influence of the hospital day type was captured in three variables: hospitalization day (expressed as a count variable); whether or not the current day was the admission day; and whether or not the 
current day was a weekend or holiday. Finally, the model accounted for admission through the emergency room (versus the clinic), hospital campus, and physician discharge practices. The latter was determined using a multi-level model to classify staff-people as either slow or fast discharge outliers (Appendix).

\section{Analysis}

Observation started at admission to the hospital and continued until patients were discharged, died, transferred to another specialty service (including the "awaiting placement unit" to which patients needing placement in long-term care are moved), or reached 31 December 2015. Patient-day formatting was used to express data so that each row in the analytical dataset represented 1 day for a particular patient. Attending inpatient physician continuity was described for each hospitalization using the "percent perfect continuity" statistic. This was calculated as the cumulative sum of the daily attending inpatient physician continuity (i.e., consecutive number of days that a patient was treated by the same physician) divided by perfect attending inpatient physician continuity (calculated as $n \times(n+1) / 2$ where $n$ is the length of stay in days).

The adjusted daily discharge probability was determined using generalized estimating equation (GEE) methods in which hospitalization days were clustered within hospitalizations which were clustered within physicians. ${ }^{8}$ Model construction started with the expected daily probability of discharge (from the TEND model) and the 1-year death probability (from the HOMR-now! model), both expressed as a log odds. Variables were kept in the model if their addition significantly decreased the Quasi-Akaike Information Statistic (QIC) statistic. ${ }^{9}$ Linear term, square root, and natural logarithm transformations of continuous variables were used to determine best fit. All continuous variables were centered on the population mean. The model used an independent correlation structure since this minimized the QIC in the base model having log odds of the discharge probability only. All analyses were conducted using SAS 9.4 (Cary, NC).

\section{RESULTS}

During the study period, there were 6301 general internal medicine admissions involving 5134 patients and 38,242 patient-days (Table 1). Patients had a mean age of 68 and were evenly divided in gender. Hospitalizations were almost equally divided between the two campuses with $85 \%$ of patients being admitted from the community (rather than long-term facilities). Comorbidities were common (the median Charlson score was 2 ) and patients had a notably high 1-year death risk (median probability of $10.4 \%$ ). The median (IQR) length of stay was 4 days $^{2-7}$ with 359 admissions (5.7\%) ending in death, 110 admissions (1.8\%) being transferred to another specialty, and $87(1.4 \%)$ patients remaining in the hospital on 31 December 2015.
Table 1 Description of Admissions to the General Internal Medicine Service in 2015

\begin{tabular}{ll}
\hline \hline & Total \\
& N=6301 \\
\hline Patient factors & \\
Mean age (SD) & $68.0(19.0)$ \\
Female & $3194(50.7 \%)$ \\
Campus & \\
- A & $3064(48.6 \%)$ \\
- B & $3237(51.4 \%)$ \\
Living status & \\
- Home & $5350(84.9 \%)$ \\
- Hospital & $161(2.6 \%)$ \\
- Nursing home & $526(8.3 \%)$ \\
- Rehab & $2(0.0 \%)$ \\
- Unknown & $262(4.2 \%)$ \\
Median Charlson score (IQR) & $2(0-3)$ \\
Median LAPS at admission (IQR) & $43(28-56)$ \\
Median expected 1-year risk death (IQR) & $10.4 \%(3.5 \%, 28.4 \%)$ \\
Admission factors & $6156(97.7 \%)$ \\
Admitted through emergency room & $3092(49.1 \%)$ \\
Came to emergency room by ambulance & $4(2-7)$ \\
Median days in hospital (IQR) & \\
Disposition & $5745(91.2 \%)$ \\
- Discharge & $359(5.7 \%)$ \\
- Died & $110(1.8 \%)$ \\
- Transferred & $87(1.4 \%)$ \\
- Remained on GIM at study end & \\
\hline
\end{tabular}

This cohort included 5134 patients and 38,242 patient-days LAPS Laboratory Abnormality Physiological Score 6

Attending inpatient physician coverage was notably fragmented. There were 41 different physicians with a median total duration of service in 2015 of 9 weeks (IQR 1.910.9 weeks). Physicians treated a median of 276 hospitalizations (IQR 123-348, range 11-627) and a median of 1035 patient-days (IQR 262-1248; range 19-2322). Changes in physician coverage were common with only slightly more than half of admissions (51.2\%) having the same attending inpatient physician throughout. Overall, there was a mean of 0.86 (SD 1.3) physician switches per admission. The mean (SD) proportion of perfect continuity was $0.78(0.24)$. There was a daily mean (SD) 17.7 (3.5) active patients on each service.

The hospital discharge model included each covariable listed in the methods section (Table 2). The daily probability of discharge increased with increases in predicted discharge odds from the TEND model (Fig. 1a). Increased severity of illness (Fig. 1b), annual death risk (Fig. 1c), and number of days in hospital (Fig. 1d) were all associated with a decreased daily discharge probability. Discharge was also less likely on the first day of admission (adjusted odds ratio [adj-OR] 0.40, 95\% CI 0.35-0.47) and on the weekend or holidays (adj-OR $0.61,95 \%$ CI $0.56-0.67$ ) (Table 2). Discharge probability differed slightly between campuses. Of the 41 attending inpatient physicians, five (12.2\%) were significantly less likely and 3 (7.3\%) were significantly more likely (after adjusting for important covariates) to discharge patients than the rest of the physicians (Appendix). The adjusted daily discharge probability was significantly influenced by these outlier physicians ("slow" dischargers adj-OR $0.82,95 \%$ CI $0.76-0.90$; "fast" dischargers adj-OR $1.41,95 \%$ CI 1.29-1.54). 
Table 2 Hospital Discharge Model

\begin{tabular}{|c|c|c|c|c|}
\hline Parameter & $\begin{array}{l}\text { Parameter } \\
\text { estimate }\end{array}$ & $\begin{array}{l}\text { Standard } \\
\text { error }\end{array}$ & $\begin{array}{l}\text { Adj-OR } \\
(95 \% \mathrm{CI})\end{array}$ & $p$ value \\
\hline Intercept & -1.5328 & 0.0328 & - & $<.0001$ \\
\hline $\begin{array}{l}\text { Discharge odds } \\
(-2.100)^{\dagger}\end{array}$ & 0.4158 & 0.0412 & - & $<.0001$ \\
\hline $\begin{array}{l}\text { HOMR-now! } \\
\text { score + } 0.79\end{array}$ & -0.0737 & 0.0089 & - & $<.0001$ \\
\hline $\begin{array}{l}\text { Daily } \\
\text { LAPS }-43^{\dagger}\end{array}$ & -0.0161 & 0.0009 & - & $<.0001$ \\
\hline Hospital day ${ }^{\dagger}$ & -0.0137 & 0.0028 & - & $<.0001$ \\
\hline $\begin{array}{l}\text { First day of } \\
\text { admission }\end{array}$ & -0.9121 & 0.0749 & $\begin{array}{l}0.40 \\
(0.35 \\
0.47)\end{array}$ & $<.0001$ \\
\hline $\begin{array}{l}\text { Weekend/ } \\
\text { holiday }^{\dagger}\end{array}$ & -0.4926 & 0.0434 & $\begin{array}{l}0.61 \\
(0.56 \\
0.67)\end{array}$ & $<.0001$ \\
\hline $\begin{array}{l}\text { Elective } \\
\text { admission }\end{array}$ & -0.4067 & 0.094 & $\begin{array}{l}0.67 \\
(0.55 \\
0.80)\end{array}$ & $<.0001$ \\
\hline Campus "A" & 0.0915 & 0.0305 & $\begin{array}{l}1.10 \\
(1.03, \\
1.16)\end{array}$ & 0.0027 \\
\hline \multicolumn{5}{|l|}{$\begin{array}{l}\text { Staff-person } \\
\text { discharge } \\
\text { practice }^{\dagger}\end{array}$} \\
\hline - Slow & -0.1936 & 0.0432 & $\begin{array}{l}0.82 \\
(0.76 \\
0.90)\end{array}$ & $<.0001$ \\
\hline - Fast & 0.3449 & 0.0450 & $\begin{array}{l}1.41 \\
(1.29, \\
1.54)\end{array}$ & $<.0001$ \\
\hline $\begin{array}{l}\text { Ln(consecutive } \\
\text { days with same } \\
\text { physician) } \\
-0.8877^{\dagger}\end{array}$ & 0.2257 & 0.0275 & - & $<.0001$ \\
\hline
\end{tabular}

The adjusted odds ratio for discharge from hospital is presented for categorical variables only. The influence of continuous covariates on the outcome is presented in Figure 1. Note that the continuous covariates are centered. Discharge odds calculated from probability of discharge from the TEND model

LAPS Laboratory Acute Physiology Score

†Time-dependent parameter

The adjusted daily discharge probability increased significantly as patient-level attending inpatient physician continuity increased (Fig. 1e). After adjusting for all other factors in the model, the daily discharge probability for the average patient increased from 15.3 to $20.9 \%$ when the consecutive number of days the patient was treated by the current physician increased from 1 to 7 days. The final model was moderately discriminative (c-statistic 0.70, 95\%CI $0.69-0.71$ ) but was very well calibrated (HosmerLemeshow $\chi^{2}$ statistic $=15.6,18 \mathrm{df}, p$ value 0.62 ) with the observed and expected daily discharge probability being very similar (Fig. 2). The mean (SD) daily discharge probability was $20.8 \%$ (11.6). Consecutive number of days that the physician had been on service was not associated with daily discharge probability independent of the factors in the hospital discharge model (Table 2).

\section{DISCUSSION}

This study found that the daily discharge probability for patients admitted to the general internal medicine service can be very precisely predicted using health administrative data typically available in hospital data warehouses. Most notably, the model found that daily discharge probability increased significantly with attending inpatient physician continuity. This finding could be considered if physician or hospital administrators wish to classify hospital resource utilization as a factor when scheduling physician coverage.

This study had several interesting findings. First, although patient factors significantly influenced the daily probability of discharge (including chronic disease burden [HOMR-now! score] and severity of illness [daily LAPS]), factors completely external to the patient (e.g., weekend/holiday status, physician discharge practice) also strongly contributed to discharge likelihood. Most relevant within this latter category was staffperson continuity with the adjusted daily discharge probability increasing for the average patient from 15.3 to $20.9 \%$ when the consecutive number of days with the same staff-person increased from 1 to 7 (Fig. 1e). Second, these data showed that patient-level attending inpatient physician continuity (i.e., the consecutive number of days each patient had with the same physician) was more tightly associated with discharge likelihood than was the consecutive number of days the physician had been on service. This suggests that the latter measure's influence on discharge probability ${ }^{4}$ is likely due to its influence on patient-specific continuity of care rather than increased physician efficiency or proficiency with increased time on service. Therefore, future studies should measure physician continuity from the patient's perspective despite this being logistically more difficult. Finally, the final model found that elective admissions had an adjusted daily discharge probability that was significantly lower than other admissions (Table 2). Most elective admissions to the general medicine service at our hospital are patients unable to function in the community who are without a new (and potentially treatable) medical issue. Such patients would await long-term care placement within the hospital and therefore have a longer length of stay.

Several issues should be noted when interpreting the study's results. First, the study examined only one patient outcome (the daily discharge probability). This is by no means the only or the most important outcome that might be influenced by physician continuity. Further studies are necessary to determine if physician continuity influences other outcomes including patient satisfaction or the likelihood of adverse outcomes such as hospital readmission, inpatient mortality, 30-day mortality, and adverse outcomes in hospital. Second, this study was limited to a single service having general internist attendings at a single center. Hopefully, these analyses can be replicated in other services and in other hospitals to determine the result's generalizability. In particular, it would be interesting to determine if these results apply to true hospitalists and non-teaching hospitals. Third, the analysis did not account for other factors that could influence the daily probability of discharge (including house-staff continuity or nursing continuity, the availability of community resources, 

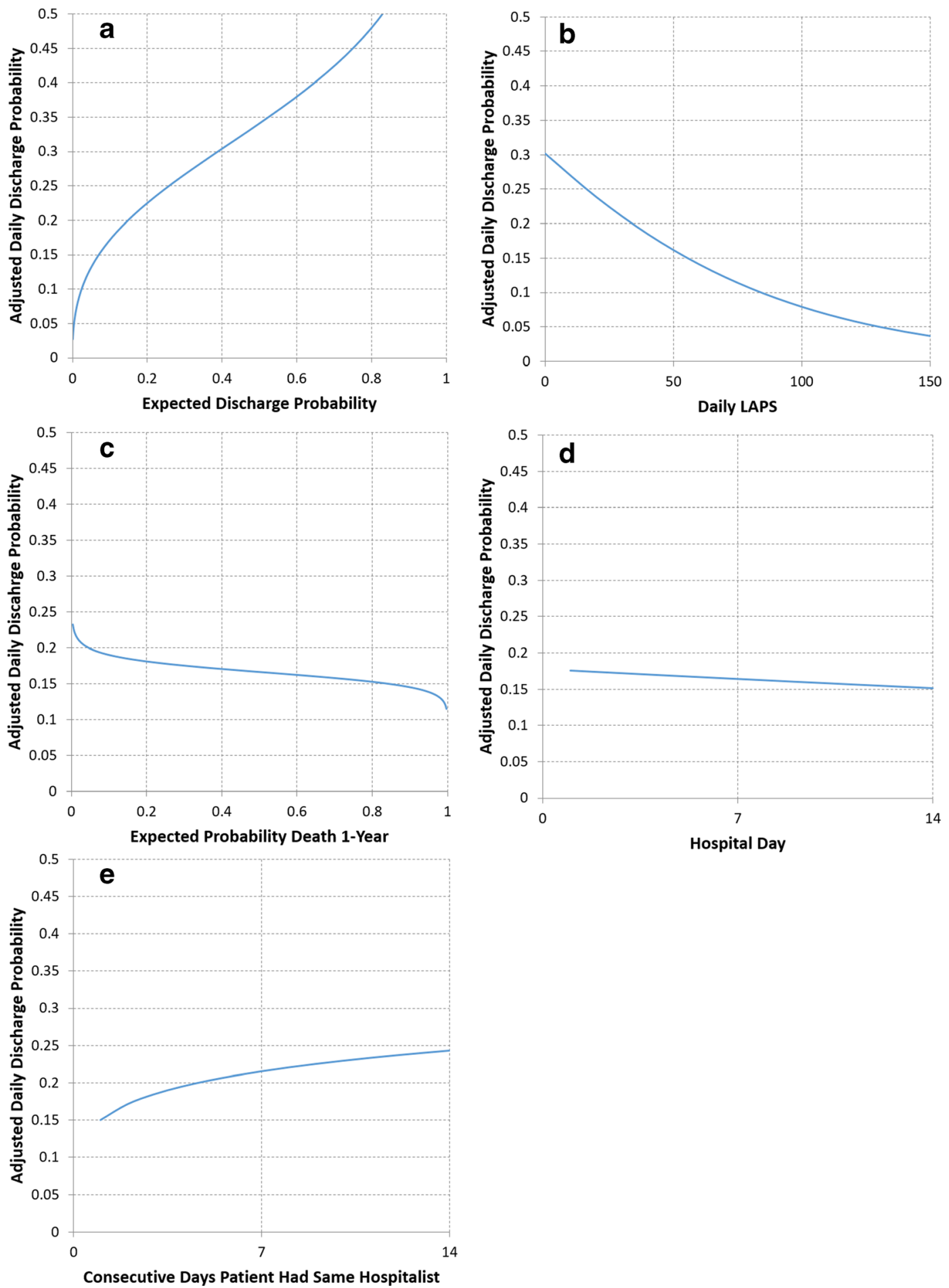

Fig. 1 a-e Independent association of expected discharge probability, daily LAPS, expected probability of death in 1 year, and staff continuity on discharge probability. Each graph plots the adjusted discharge probability against one of the continuous variables in the final model (Table 2). These associations are adjusted for each other as well as the categorical covariates whose influence on the outcome is presented in Table 2. The adjusted discharge probabilities presented here are those for a patient-day with reference values for all binary covariates and average values for all continuous variables. LAPS Laboratory Abnormality Physiological Score ${ }^{6}$.

familial support, etc.). This might explain why the final hospital discharge model (Table 2) had mediocre discrimination (c-statistic 0.7 ) despite having excellent calibration (Fig. 2). Perhaps the discrimination of future daily discharge probability models could be improved by considering these and other factors. Finally, while the model presented here (Table 2) showed that patient-level attending inpatient physician continuity was significantly and independently associated with daily discharge probability, we could not quantify how defragmenting physician coverage (i.e., rearranging attending inpatient physician coverage so that the number of patient hand-overs between 


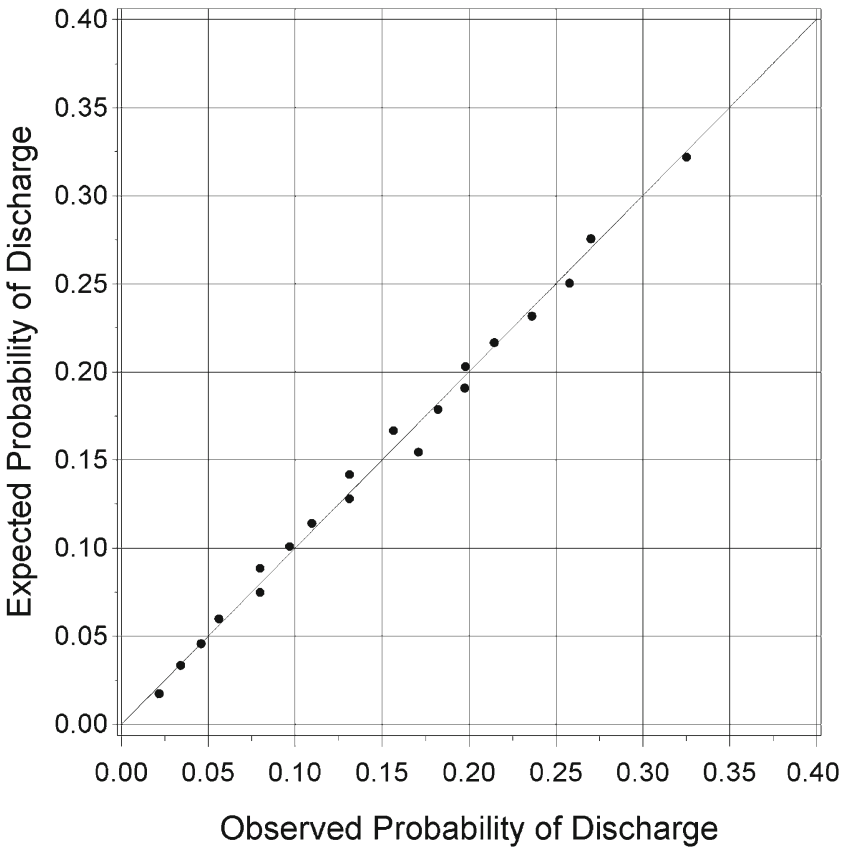

Fig. 2 Observed versus expected daily hospital discharge probability. The expected daily probability of discharge was generated using the hospital discharge model (Table 2). The expected probabilities did not differ significantly from observed (Hosmer-Lemeshow $\chi^{2}$ statistic $=11.5,18 \mathrm{df}, p$ value 0.87 ).

physicians is minimized) would influence hospital length of stay. This will likely require a different modeling technique perhaps with Monte Carlo methodologies.

In conclusion, increased attending inpatient physician continuity independently increased the likelihood that patients were discharged from hospital. Further research is needed to determine the generalizability of this result and the influence of physician continuity on other outcomes.

Corresponding Author: Carl Walraven, MD MSc FRCPC; Medicine and Epidemiology \& Community Medicine, University of Ottawa, ICES uOttawa, Ottawa Hospital Research Institute, Ottawa, ON, Canada (e-mail: carlv@ohri.ca).

Funding Information This study was supported by the Department of Medicine, University of Ottawa.

\section{Compliance with Ethical Standards:}

Conflict of Interest: The author declares no conflicts of interest.

\section{REFERENCES}

1. Ali NA, Hammersley $\mathbf{J}$, Hoffmann $\mathbf{S P}$ et al. Continuity of care in intensive care units: a cluster-randomized trial of intensivist staffing. Am J Respir Crit Care Med 2011;184:803-808.

2. Epstein $\mathbf{K}$, Juarez E, Epstein A, Loya $\mathbf{K}$, Singer A. The impact of fragmentation of hospitalist care on length of stay. J Hosp Med 2010;5:335-338.

3. Blecker S, Shine D, Park $\mathbf{N}$ et al. Association of weekend continuity of care with hospital length of stay. Int J Qual Health Care 2014;26:530-537.

4. van Walraven $\mathbf{C}$. The influence of hospitalist continuity on the likelilhood of patient discharge in general medicine patients. J Hosp Med 2018; 13:E1-E3.

5. van Walraven C, Forster AJ. The TEND (Tomorrow's Expected Number of Discharges) model accurately predicted the number of patients who were discharged from the hospital in the next day. J Hosp Med. 2018; 13: 158163.

6. Escobar GJ, Greene JD, Scheirer P, Gardner MN, Draper D, Kipnis P. Risk-adjusting hospital inpatient mortality using automated inpatient, outpatient, and laboratory databases. Med Care 2008;46:232-239.

7. van Walraven C, Forster AJ. HOMR-now! A modification of the HOMR score that predicts 1-year death risk for hospitalized patients using data immediately available at patient admission. Am J Med. 2017; 130(8): 991.e9-991.e16.

8. Stokes ME, Davis CS, Koch GG. Generalized estimating equations. Categorical Data Analysis Using the SAS System. 2nd ed. Cary, NC: SAS Institute Inc; 2000;469-549.

9. Pan W. Akaike's information criterion in generalized estimating equations. Biometrics 2001;57:120-125.

Publisher's Note Springer Nature remains neutral with regard to jurisdictional claims in published maps and institutional affiliations.

\section{CLASSIFYING ATTENDING INPATIENT PHYSICIAN DISCHARGE PATTERNS}

This was determined using a multi-level logistic regression model in which patient-days were grouped within attending inpatient physicians. Attending inpatient physician identification was treated as random effects with all other covariates that were included being treated as fixed effects. Method was Laplace, DDFM was BW, and TYPE was CS. Attending inpatient physicians whose random intercept was significantly lower than the population mean were classified as "Slow Dischargers" (red markers); those whose intercept was significantly greater than the population mean were classified as "Fast Dischargers" (blue markers). 